\title{
Almacenamiento de carbono en arreglos agroforestales asociados con café (Coffea arabica) en el sur de Colombia
}

\section{Carbon storage in agroforestry arrangements associated with coffee (Coffea arabica) in the south of Colombia}

\author{
Armazenamento de carbono em arranjos agroflorestais \\ associados com café (Coffea arabica) no sul da Colômbia
}

\author{
Gustavo Vega Orozco ${ }^{1}$, Claudia Mercedes Ordoñez Espinosa², \\ Juan Carlos Suarez Salazar ${ }^{3}$ \& César Fabián López Pantoja ${ }^{4}$ \\ 'Ingeniero agrónomo, Magister en Ciencias Agrícolas, Magister en Sistemas Sostenibles de Producción. \\ ${ }^{2}$ Ingeniera Agropecuaria, Especialista técnica en evaluación de la calidad del café, Magister en Sistemas \\ Sostenibles de Producción. ${ }^{3}$ Ingeniero Agroecólogo, Especialista técnico en evaluación de la calidad del café, \\ Magister Scientiae Agroforesteria Tropical, estudiante Doctorado en Ciencias-Biología línea \\ Ecofisiologia en la Universidad Nacional de Colombia. ${ }^{4}$ Ingeniero Agroforestal, \\ Especialista técnico en evaluación de la calidad del café \\ 1,2,4Servicio Nacional de Aprendizaje SENA -Centro de Gestión y Desarrollo Sostenible \\ Surcolombiano Regional Huila, Colombia. \\ ${ }^{3}$ Universidad de la Amazonia. Facultad de Ingeniería \\ 'gvega60@misena.edu.co, ${ }^{2} \mathrm{clmechas} 24 @$ misena.edu.co, \\ 3juansuarez1@gmail.com, ${ }^{4}$ ingcesar@misena.edu.co.
}

\section{Resumen}

Se calculó la cantidad de carbono almacenado en cuatro tipologías de sombra en sistemas cafeteros del sur de Colombia: Alta densidad de árboles con sombra diversificada (Alta.Dens), Guamo en alta densidad (Guam.Ad), Nogal en alta densidad (Nog.Ad), Plátano con baja diversidad (Plat. Bdiv), Sombra compleja diversificada (Com.Div), mediante el uso de ecuaciones alométricas para medición biomasa; el estudio se desarrolló entre Junio y Diciembre de 2012. Las tipologías con mayor tasa de almacenamiento de carbono fueron: Com.Div y Guam.Ad con 87,37 y $58,11 \mathrm{t} \mathrm{ha}^{-1}$ de $C$ respectivamente. Las tipologías Alta.Dens y Guam.Ad presentaron menor tasa de almacenamiento de carbono. Se encontró relación entre el $\%$ de cobertura de las tipologías y el aumento en la acumulación de carbono; es decir, los sistemas con mayor diversificación presentaron mayores valores de acumulación de carbono.

Palabras Clave: agroecosistemas cafeteros, cambio climático, ecuaciones alométricas, diversidad de árboles.

\section{Abstract}

It was calculated the carbon amount stored in four types of shade coffee systems in the south of Colombia: high density of trees for diversified shade (Alta. Dens), Guamo in high density (Guam.Ad), Walnut in high density (Nog.Ad), Banana with low diversity (Plat.bdiv), Complex diversified shadow (Com.Div), through the use of allometric equations for measuring biomass; the study was developed between June and December of 2012. The 
typologies with the highest rate of carbon storage were: Com.Div and Guam.Ad with 87.37 and 58.11 $\mathrm{t}$ ha ${ }^{-1}$ of $\mathrm{C}$ respectively. The typologies Alta. dens and Guam.Ad had a lower rate of carbon storage. A relation between the \% of coverage of the typologies and the increase in the accumulation of carbon was found; that means, systems with higher diversification presented higher values of carbon buildup

Key-words: coffee agroecosystems, climate change, allometric equations, diversity of trees

\section{Resumo}

Calculou-se a quantidade de carbono armazenado em quatro tipologias de sombra em sistemas cafeteiros do sul da Colômbia: Alta densidade de árvores com sombra diversificada (Alta.Dens)
Guamo em alta densidade (Guam.Ad), Nogal em alta densidade (Nog. Ad), Banana com baixa diversidade (Plat.Bdiv) Sombra complexa diversificada (Com.Div) usando equações alométricas para a medição da biomassa; O estudo foi realizado entre Junho e Dezembro de 2012. Tipologias com a maior taxa de armazenamento de carbono foram Com.Div e Guam.Ad com 87,37 e 58,11 t ha-1 of C, respectivamente. Os tipos Alta.Dens e Guam.Ad tiveram menor taxa de armazenamento de carbono. Foi encontrada relação entre \% de cobertura das tipologias e aumento no acúmulo de carbono; assim, sistemas mais diversificados apresentaram maiores valores de acúmulo de carbono.

Palavras-chave: agroecossistemas de café alterações climáticas, equações alométricas, diversidade de árvores.

\section{Introducción}

Desde 1950, los seres humanos han transformado significativamente los ecosistemas debido a las demandas crecientes de alimentos, agua dulce, madera, fibra y combustible (Millennium Ecosystem Assessment, 2005). El modelo agrícola industrial, basado en el uso de las tecnologías modernas, contribuye significativamente con la emisión de gases con efecto invernadero como el metano, óxido nitroso y dióxido de carbono (Goncalves, 2008; Novoa et al., 2000), los cuales provocan el incremento de su concentración en la atmósfera. (Ortiz et al., 2008). El dióxido de carbono (CO2) es el gas que más contribuye $(573,5$ Gg año-1) al calentamiento global; para mitigar los efectos causados por estos gases existen alternativas como el almacenamiento de los mismos en la biomasa arbórea (mediante la fotosíntesis) y en el suelos a través de la acumulación de materia orgánica (Ávila et al., 2001; Segura et al., 2002).

Según lo planteado la agroforestería se convierte en una estrategia importante para el almacena- miento y captura de Carbono, la biodiversidad de especies arbóreas con potencial para ser asociadas en diferentes diseños agroforestales por ejemplo bosque protectores, barreras rompe vientos, arboles dentro de los cultivos (Farfán, 2012), utilizados en programas de forestación y reforestación (Kumar et al., 2011; Montagnini, 2004; Ruark et al., 2003). El potencial de captura y almacenamiento de carbono en sistemas agroforestales (12 y 228 ton $^{-1} \mathrm{C} \mathrm{ha}^{-1}$ ) (Andrade et al, 2003), se debe a que el carbono (elemental), depositado en follaje, tallos, sistema radicular y principalmente en tejido leñoso de troncos y ramas de los árboles (Pérez et al., 2005). El flujo y captura de carbono en la biosfera terrestre ha incrementado el interés global en estos usos de suelo como opción para estabilizar las emisiones de gases de efecto invernadero (Dixon, 1995; Suarez, 2002).

Actualmente el secuestro de carbono mediante los bosques se ha convertido en un servicio ecosistémico reconocido a escala global, el cual puede 
ser valorado económicamente. Ramírez et al. (1999). En la cumbre de Rio de Janeiro se definió como mecanismo económico y legal para la protección de los ecosistemas a los Pagos por Servicios Ambientales (PSE), como instrumento con el cual se valoran los servicios del ecosistema, mediante Mecanismos de Desarrollo Limpio (MDL) que incentivan la reforestación en países en vía de desarrollo para cumplir el compromiso de control de emisiones por parte de países desarrollados.

El desarrollo mundial de la industria de cafés especiales (por ejemplo café de sombra) es creciente, un mercado para este tipo de café es EE.UU donde se ha estimado un valor de venta \$ 30-32 billónes de dólares, aproximadamente un volumen de participación del 37\% (Specialty Coffee Association of America - SCAA, 2012). Un café se considera especial cuando es percibido y valorado por los consumidores por alguna característica que lo diferencia de los cafés convencionales por lo cual están dispuestos a pagar un precio superior (Fedecafé, 2012), este tipo de mercados se han convertido en una alternativa a la actual crisis cafetera que afronta el sector a nivel mundial (Lozano 2009; Ordoñez 2013). Sin embargo la producción de cafés de especialidad es la suma de factores como las condiciones agroecológicas y de manejo, caso especial los cafés producidos en sistemas asociados con árboles, los cuales pueden generar ingresos adicionales por medio de los pagos por servicios ambientales. Ante esta nueva perspectiva, el objetivo del presente trabajo fue calcular la cantidad de carbono almacenado en arreglos agroforestales asociados con café en el sur de Colombia.

\section{Materiales y Métodos}

\section{Descripción del área de estudio}

El presente estudio se desarrolló en el departamento de Huila, municipio de Pitalito ubicado a los

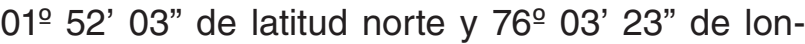

gitud oeste. La zona de vida según clasificación de Holdridge corresponde a bosque húmedo premontano (bh-PM), altura entre 1.200 a 1.500 m, temperatura entre 18 y 24 oC, precipitación total anual media de $1.200 \mathrm{~mm}$ (Espinal et al., 1997). Las características ubican al municipio en el Ecotopo cafetero 319ª (Gómez et al., 1991).

\section{Inventario forestal}

Se realizó un censo de árboles (con dap $>10 \mathrm{~cm}$ ) en 30 parcelas de café de cinco tipologías de sombra: a) Plátano con baja diversidad (Plat.Bdiv), b) Alta densidad de árboles con sombra diversificada (Alta.Dens), c) Nogal en alta densidad (Nog. Ad), d) Guamo en alta densidad (Guamo.Ad) y e) Sombra compleja diversificada (Com.Div), el criterio para definir las tipologías de la sombra fue la composición botánica, donde se consideraron las 4 especies de plantas más abundantes asociadas al cultivo de café según Deheuvels et al. (2012). Se creó una base de datos con variables dasométricas de cada individuo: altura, altura comercial, diámetro a la altura de pecho $(1,3 \mathrm{~m})$ y tres diámetros de copa (Schlegel et al., 2002), los árboles fueron georreferenciados con dispositivo GPS Garmin Etrex HCX, luego los datos se descargaron en el software DNRGPS $6.0 \AA$ y procesados en el software ArcGIS $10 \circledR$.

\section{Estimación de carbono almacenado en biomasa}

Se estimó la biomasa de los individuo mediante la ecuación alométrica propuesta por Chave et al. (2005) para bosques tropicales y especies individuales, donde se utilizan las variables dap y densidad de la madera por especie (Zanne et al., 2009). Los valores de biomasa se llevaron a lote y a total por tipología.

\section{Cálculo de carbono por tipología}

El carbono total almacenado se calculó al llevar la biomasa total a unidades de carbono mediante la ecuación (1) propuesta por Segura et al. (2002): 
$\mathrm{CA}=\mathrm{B}{ }^{*} \mathrm{FC}$

(CA): Carbono almacenado, (t ha-1 de C)

(B): Biomasa total (t ha-1 de C)

(FC): Fracción de carbono que en este caso se utilizó 0,5 establecido por el Panel Intergubernamental de Cambio climático (IPCC).

\section{Porcentaje de cobertura por tipología}

Para el cálculo de la cobertura por tipología se determinó el área de copa de cada individuo a partir de los diámetros de copa. Se creó una base en el software ArcGIS $10 \AA$ con las variables dasométricas para posteriormente generar los Buffer de copa con el área promedio como unidad de medida.

\section{Análisis estadístico}

Se utilizó el programa estadístico InfoStat V (2012) y se aplicó un análisis de varianza junto con la prueba de comparación LSD Fisher para determinar las diferencias en el almacenamiento de carbono por tipología evaluada.

\section{Resultados}

\section{Inventario forestal}

Durante el inventario forestal se registraron 38 especies, 22 familias y 1136 individuos, la familia Fabaceae fue la más representativa con 10 especies $(50,48 \%)$, en su mayoría individuos Inga sp. (349), y Erythrina poeppigiana (142); la segunda familia en orden de importancia $(9,64 \%)$ fue Boraginaceae (112 individuos), al cual pertenece el Nogal cafetero (Cordia alliodora), las familias de menor representación fueron Musaceae (Musa spp.) y Lauraceae (Persea americana) con $8,43 \%$ y $7,14 \%$ respectivamente; la familia Bignoniaceae, Myrtaceae, Rutaceae aportaron cada una 3 especies (7,14\%) (Figura 1). Autores como Farfán et al. (2003), reporta en Colombia especies comúnmente empleadas como componente arbóreo en los sistemas agroforestales con café pertenecen a la familia Fabaceae, especialmente Inga sp. Albizzia sp., Erythrina sp y Leucaena leucocephala, datos similares a los encontrados en la presente investigación.

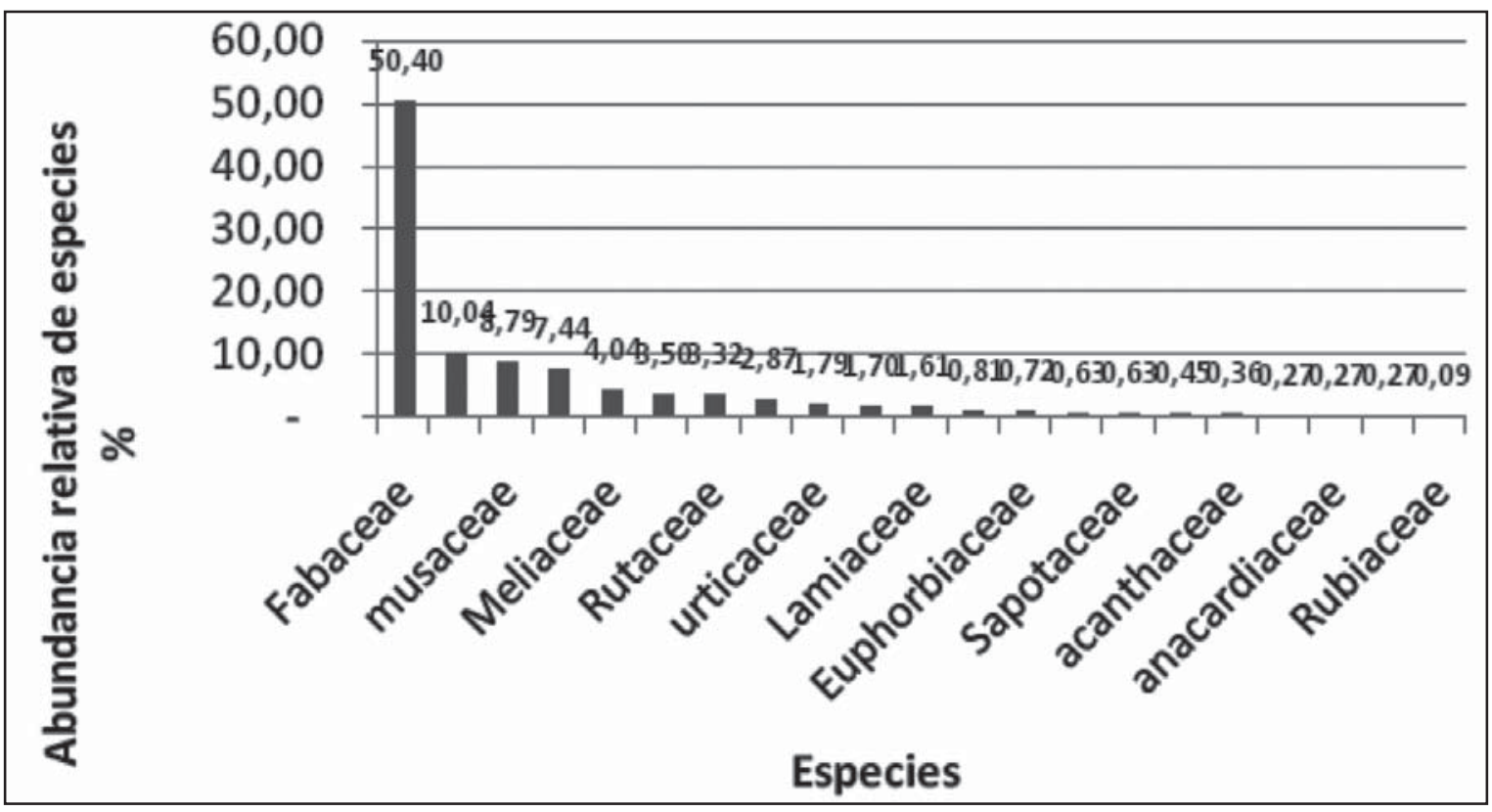

Figura 1. Distribución de familias de árboles en el área de estudio.

\section{Estructura vegetativa por tipología de sombra}

La Tabla 1, indica que la tipología Alta.Dens presento los mayores valores en las variables altura promedio $(9,59 \mathrm{~m})$, cobertura arbórea $(55,69 \%)$ y densidad (87,65 individuos. ha $\left.{ }^{-1}\right)$; entre tanto la 
tipología Guam.Ad registro el mayor dap (19,05 $\mathrm{cm})$ y área de copa promedio (17,1 $\left.41 \mathrm{~m}^{2}\right)$; Nog. Ad mostró los mayores valores en área basal promedio y máxima $\left(4,62\right.$ y $8,41 \mathrm{~m}^{2} \mathrm{ha}^{-1}$ respecti- vamente). Plat.Bdiv y Com.Div no registraron valores superiores en ningunas de las variables de estructura vegetativa.

Tabla 1. Estructura vegetativa por tipología de sombra

\begin{tabular}{lccccc}
\hline \multicolumn{1}{c}{ Variables } & Alta.Dens & Guam.Ad & Nog.Ad & Plat.Bdiv & Com.Div \\
\hline $\begin{array}{l}\text { Diámetro a la altura de pecho } \\
\text { promedio }(\mathrm{cm})\end{array}$ & 13,71 & 19,05 & 17,76 & 6,13 & 14,09 \\
Área basal promedio $\left(\mathrm{m}^{2} \mathrm{ha}^{-1}\right)$ & 0,7 & 1,03 & 4,62 & 0,94 & 1,21 \\
Área basal promedio máximo $\left(\mathrm{m}^{2} \mathrm{ha}^{-1}\right)$ & 1,84 & 2,8 & 8,41 & 2,21 & 4,92 \\
\hline Altura promedio $(\mathrm{m})$ & 9,59 & 6,32 & 8,02 & 3,15 & 2,97 \\
\hline Altura comercial promedio $(\mathrm{m})$ & 3,2 & 1,9 & 4,96 & 1,34 & 3,16 \\
\hline Área de copa promedio $\left(\mathrm{m}^{2}\right)$ & 14,2 & 17,1 & 4,23 & 5,18 & 16,32 \\
\hline Cobertura arbórea $(\%)$ & 55,69 & 50,16 & 13,23 & 12,07 & 42,86 \\
\hline Densidad (individuos ha-1 $)$ & 87,65 & 8,57 & 10,11 & 11,96 & 14,52 \\
\hline
\end{tabular}

El comportamiento de la tipología Alta.Dens en las variables estructurales altura promedio cobertura arbórea y densidad está relacionado con la presencia de especies como Guamo (Inga sp.), E. poepigiana y Nogal (Cordia alliodora), además del número de especies y densidad de individuos en mayor cantidad por hectárea $(10,71 \pm 1,71$ y 87,65 respectivamente) comparada con las otras tipologías.

Para la tipología Guam.Ad la variable cobertura presentó el segundo mayor valor dentro del muestreo, esto se debe a las condiciones morfológicas de copa amplia (17 m) y alta cantidad de biomasa que presenta la especie Guamo (Inga $s p$ ). Ante esto Farfán et al. ( 2010) reporta que Guamo es un árbol de copa densa, ancha, aparasolada y ramificada utilizado ampliamente como sombrío para cafetales, además, por su aporte significativo de materia orgánica y mejoramiento de las características físicas del suelo. Para la variable dap, la tipología presenta el mayor valor (19,05 m) posiblemente debido a la edad de los árboles del muestreo y a que generalmente son individuos sin algún tipo de manejo silviucltural

\section{Biomasa y carbono por tipología de sombra}

Las tipologías con mayor cantidad de biomasa y carbono almacenado fueron Com.Div y Guam. Ad con 84,37 y 58,11 t ha-1 de C (Figura 2). Los datos de la presente investigación son similares para Com.Div donde predominan las especies de Cachingo (E. poepigiana), respecto a lo encontrado por Albrecht et al. (2003) con 30 y $55 \mathrm{t} \mathrm{ha}^{-1}$ año-1 $\mathrm{C}$ almacenado e inferiores a lo reportado por Ávila et al. (2001) en la misma especies donde se almacenó $195 \mathrm{t} \mathrm{ha}^{-1}$. En la tipología Guam. $\mathrm{Ad}$ el valor es superior a lo reportado por Suarez (2002) para café productivo en abandono (CPAB) compuesto por sombríos de Guamo (Inga sp.) y Laurel (Cordia alliodora) con 27,3 t ha-1 $\mathrm{C}$ y en café productivo con especies maderables se encontró $31,8 \mathrm{t} \mathrm{ha}{ }^{-1} \mathrm{C}$. 


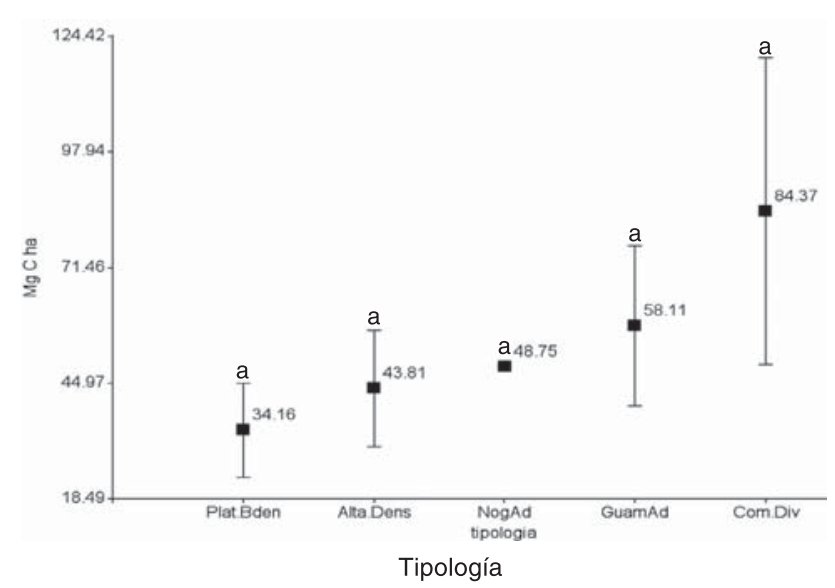

Figura 2. Cantidad de carbono almacenado por tipología según prueba de LDS Fisher $(\mathrm{P}<0.05)$.

El comportamiento en almacenamiento de carbono de las tipologías Alta.Dens, Nog.Ad, Guam.Ad y Com.Div (Figura 2) son superiores a lo reportado por Albrecht et al. (2003) en sistemas agroforestales con $95 \mathrm{t} \mathrm{ha}^{-1}$ de C, Para el mismo sistema Dixon et al. (1993) reporta un potencial de captura de carbono de $34 \mathrm{t} \mathrm{ha}^{-1}$ de $\mathrm{C}$, Según Schroeder
(1994) el almacenamiento de carbono en sistemas agroforestales de regiones semiáridas, subhúmedas, húmedas y templadas oscila entre 9, 21, 50, y 63 t ha $^{-1}$ de $\mathrm{C}$, respectivamente, datos que se encuentran en el rango de las tipologías analizadas. (34,16 a 84,37 Mg C ha-1) (Figura 2). La tipología Plat.Bdiv almacenó $34,16 \mathrm{t} \mathrm{ha}^{-1}$ de $\mathrm{C}$ superior a lo reportado por Brakas et al. (2011) en Filipinas para sistemas multiestrato de banano (Musa spp.) + Árboles frutales los cuales almacenaron en biomasa aérea $13,6 \mathrm{t} \mathrm{ha}^{-1}$ de $\mathrm{C}$.

Diferentes autores afirman que variaciones en la acumulación de carbono en biomasa de plantas para sistemas agroforestales dependen de la variación de clima, tipo de suelo, sistema de manejo de los árboles, diseño (densidad de árboles) y especies de árboles utilizados (Kang, 1993; Evensen et al. 1994; Viswanath et al., 1998; Kang et al., 1999; Albrecht et al., 2003; Oelbermann et al. 2004).

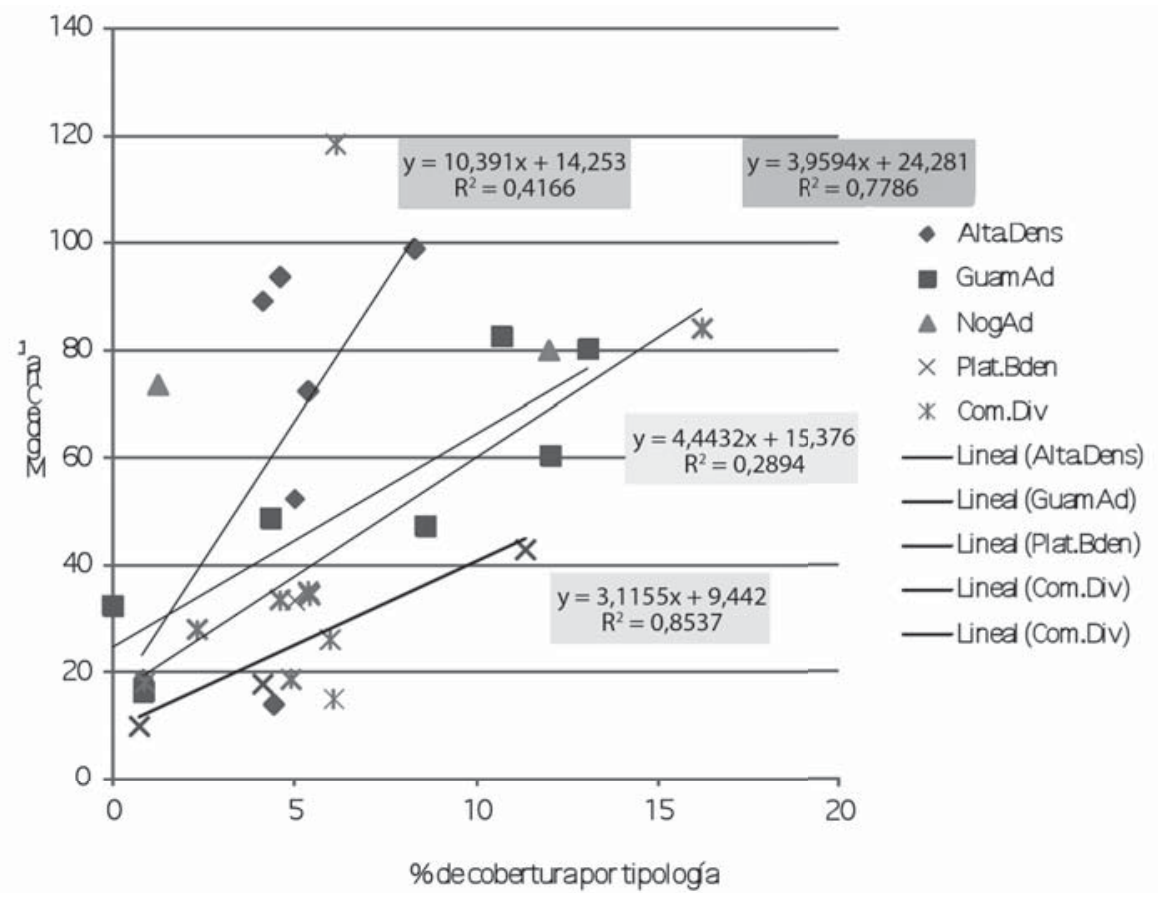

Figura 3. Relación entre carbono almacenado y el porcentaje de cobertura por tipología. 


\section{Discusión}

En sistemas cafeteros con arreglos agroforestales anteriormente se usaban más de 100 especies de árboles para sombra Beer et al. (1998), para el caso de Colombia se observa un menor número de especies de sombrío en los cafetales, en el que se destaca el Guamo (Inga sp.) como especie empleada en la zona cafetera (Cardona et al., 2005); la presencia de esta especie en la zona de estudio se puede relacionar con procesos de certificación cafetera, en los cuales el porcentaje de sombra debe ser mínimo $40 \%$ y estar dominada por una o dos especies entre ellas el Inga sp. (Farfán, 2012). Factores como el rápido desarrollo vegetativo, tolerancia a suelos ácidos, adaptarse a amplios rangos altitudinales, alta producción de materia seca $(6,0$ ton ha-1 año-1) (Farfán, 2007), reciclaje de nutrientes, control de arvenses y erosión de los suelos (Farfán et al., 2010; Instituto biológico Alexander Von Humboldt, 2013), son tenidos en cuenta en el momento de asociar Inga sp. a los sistemas cafeteros en la zona de estudio.

El cachimbo (E. poeppigiana) es otra de las especies con presencia en la zona de estudio; según Escobar (2007) en sistemas cafeteros del departamento del Huila, este género es el más utilizado para asociar al cultivo de Coffea arabica, según Cárdenas (2008) en Costa Rica esta especie es utilizada por sus múltiples beneficios: fijación de nitrógeno, aporte de biomasa, leña, entre otros.

La existencia de nogal (Cordia alliodora) en sistemas cafeteros de la presente investigación se debe a características dasométricas de copa pequeña, estrecha, abierta y su relación con la Radiación Fotosintéticamente Activa (RAFA), dado que la forma de la copa pueden afectar la transmisión de luz (Dzib, 2003), entre otras características esta su rápido crecimiento vegetativo, poda natural y producción de madera de calidad para las industrias del mueble y la ebanistería (Farfán, 2004). Según Cárdenas (2008) en Costa Rica es la segunda especie para sombra en sistemas cafeteros, por su facilidad para la regeneración, establecimiento, asociación con otros cultivos, alto valor de la madera para uso en la finca, comercio local y mercados nacionales.

\section{Principales usos de las especies agroforestales asociadas al café}

La implementación de especies dentro de los arreglos con $\mathrm{C}$. arabica cumple propósitos ecológicos y funcionales como alimentación, leña y madera. Según Perfecto (1996) en Centroamérica, es común encontrar plátano (Musa spp.) cítricos (por ejemplo Citrus nobilis, Citrus aurantium y Citrus limón), u otros árboles frutales (por ejemplo Persea americana y Mangifera indica) mezclado con $C$. arabica, así como algunos árboles productores de madera y leña, como $C$. alliodora. En Costa Rica la estructura típica de sistemas agroforestales con $C$. arabica está compuesta por arboles de sombra como E. poeppigiana, una especie productora de frutas Musa spp., Mangifera indica, Inga sp. y un estrato superior de árboles maderable como Cedrela odorata y C. alliodora (Lagemann et al., 1983).

Lo reportado por los anteriores autores es similar a lo encontrado en el presente estudio donde se identificaron además de especies con potencial dendroenergético $(54,4 \%)$ como el Inga sp. y Psium guajaba, especies para madera como Cedrela odorata, Eucalyptus globulus, y productoras de frutas como naranja (Citrus sinnensis), mandarina (Citrus nobilis) y limón (Citrus limón).

En el estudio se determinó el \% de cobertura por tipología y su relación con el almacenamiento de carbono en la biomasa aérea (Figura 3). En general, el valor máximo de carbono se encontró en la tipología Com.Div (84,37 t ha-1 de C ) con 42,86\% cobertura arbórea, la mínima cantidad de carbono almacenada 34,16 t ha-1 de $\mathrm{C}$ la presentó la tipología Plat.Bdiv con $12,07 \%$ cobertura. 
Es necesario considerar que las tasas de almacenamiento de carbono dependen de la edad y densidad de plantas, fertilidad, tipo de suelo y características del sitio (factores climáticos) y el manejo silvicultural al que se vea sometido el SAF (Brakas et al., 2011; Dzib, 2003). Según Haggar et al. (2013) en Guatemala los sistemas de producción de $C$. arábiga se encuentran asociados con árboles de Inga $s p$., y pueden almacenar $39 \mathrm{t} \mathrm{ha}^{-1}$ de $\mathrm{C}$ cuando existe alta diversidad de especies nativas asociadas ( $7-12$ especies por hectárea), datos superiores a los encontrados en esta investigación.

\section{Conclusiones}

Los arreglos agroforestales asociados al cultivo de café en el sur de Colombia presentaron un alto número de especies (2 a 10), representadas por Inga sp., plátano (Musa spp.) y Cachimbo (E. poepigiana), usadas por su morfología, rápido desarrollo vegetativo y diferentes servicios prestados como sombrío, leña, madera o frutales.

La presencia de las especies de mayor dominancia en los sistemas agroforestales estudiados se deben a los procesos de certificación y las políticas de la Federación Nacional de Cafeteros.

La incorporación de árboles a sistemas de producción de café en una estrategia para la generación de servicios ecosistémicos en el marco de mecanismos de desarrollo limpio, que pueden ser incentivados a través de bonos solidarios de carbono.

\section{Agradecimientos}

A los caficultores del municipio de Pitalito por brindarnos su información, al comité municipal de cafeteros por su valiosa colaboración y demás personas que de una u otra forma realizaron sus aportes a la investigación.

\section{Literatura citada}

1. Ávila, G., Jiménez, F., Beer, J., Gómez, M. \& Muhammad., I. (2001). Almacenamiento, fijación de carbono y valoración de servicios ambientales en sistemas agroforestales en Costa Rica. En Agroforestería en las Américas Vol 8 No 30. PP. 32 - 35.

2. Andrade, J. \& Ibrahim, M. (2003). ¿Cómo monitorear el secuestro de carbono en sistemas silvopastoriles? En: Revista Agroforestería de las Américas vol. 10. p 1-8.

3. Albrecht, A. \& Kandji, S.T. (2003). Carbon sequestration in tropical agroforestry systems. In: Agriculture, Ecosystems and Environment 99:15-27.

4. Beer, J., Muschler, R., Kass, D. \& Somarriba, E. (1998). Shade management in coffee and cacao plantations. Agroforestry Systems 38:139-164.

5. Brakas, S.G. \& Aune, J.B. (2011). Biomass and Carbon Accumulation in Land Use Systems of Claveria, the Philippines In: Carbon Sequestration Potencial of Agroforestry Systems, Advances in Agroforestry, Volumen 8, 163 - 165. Norway.

6. Cardona, D. A. \& Sadeghian, S. (2005). Beneficios del sombrío de guamo en Suelos cafetero. Avances técnicos CENICAFE No. 335: 8 p. Colombia.

7. Cardenas, A.D. (2008). Incentivos económicos para la producción ecoamigable en fincas cafetaleras en el Corredor Biológico Volcánica Central - Talamanca, Costa Rica. Tesis M.Sc. CATIE. Turrialba, Costa Rica 200 p.

8. Deheuvels, O., Avelino, J., Somarriba, E. \& Malezieux, E. (2012). Vegetation structure and productivity in cocoa-based agroforestry systems in Talamanca, Costa Rica. In: Agriculture, Ecosystems and Environment 149:181- 188.

9. Dixon, R.K., Winjum, J.K. \& Schroeder, P.E. (1993). Conservation and sequestration of carbon: the potential of forest and agroforestry management practices. Glob. Environ. Change 3, 159-173.

10. Dixon, R.K. (1995). Agroforestry systems: sources or sinks of greenhouse gases? Agrofor. Syst. 31

11. Dzib, B.B. (2003). Manejo, secuestro de carbono e ingresos de tres especies forestales de sombra en cafetales de tres regiones contrastantes de Costa Rica. Maestría en Agroforestería Tropical

12. Evensen, C.I., Dierolf, T.S. \& Yost, R.S. (1994). Growth of four tree species managed as hedgerows in response to liming on acid soil in west Sumatra, Indonesia. Agrofor. Syst. 27, 207-222.

13. Escobar, G. (2007). El sombrío en los cafetales: un sistema, una estrategia para la seguridad alimentaria p. 258 - 296.Recuperado de: http://www.academia. edu/630746/El_sombrio_en_los_cafetales_un_sistema_una_estrategia_para_la_seguridad_alimentaria.

14. Farfán, V. F. (2012). Arboles con potencial para ser incorporados en sistemas agroforestales con café. Chinchiná (Colombia): Cenicafe - Fondo Nacional del Café, 
15. Farfán V., F., Baute B. \& J. E. (2010) Efecto de la distribución espacial del sombrío de especies leguminosas sobre la producción de café Cenicafe 61(1):35-45

16. Farfán V, F., Baute B, J.E., Sánchez A, P.M. \& Menza Franco, H.D. (2010). Guamo santafereño en sistemas agroforestales con café. Avances Técnicos - CENICAFE No 396, 1 - 8. Colombia.

17. Farfán V. F. (2007). Producción de café en Sistemas Agroforestales. En: Sistemas de producción de café en Colombia. Chinchiná, Cenicafe. pp. 161 - 200

18. Farfán V., F. \& Urrego, J.B. (2004). Comportamiento de las especies forestales Cordia alliodora, Pinus oocarpa y Eucalyptus grandis como sombrío e influencia en la productividad del café. Cenicafe 55(4):317 - 329.

19. Farfán, V.F., Arias H, J.J. \& Riaño H, N.M. (2003). Desarrollo de una metodología para medir sombrío en sistemas agroforestales con café. Cenicafe 54(1):24-34

20. Federación Nacional de Cafeteros de Colombia FEDECAFE (2012). Precio Interno del café Colombiano. (En línea). Recuperado de: www.federaciondecafeteros.org/static/files/Precio_interno_diario.xls

21. Gómez, L., Caballero, A. \& Baldión, J.V. (1991). Ecotopos cafeteros. Federación Nacional de Cafeteros de Colombia. Santafé de Bogotá D.C. 125 Pág.

22. Kang, B.T., Caveness, F.E; Tian, G \& Kolawole, G.O. (1999). Long-term alley cropping with four species on an Alfisol in southwest Nigeria-effect on crop performance, soil chemical properties and nematode population. Nutr. Cycl. Agroecosyst. 54, 145-155.

23. Kang, B.T. \& (1993). Alley cropping: past achievements and future directions. Agrofor. Syst. 23, 141-155.

24. Kumar B, M., Nair P, .K.R. Ramachandran (2011). Carbon Sequestration Potencial of Agroforestry Systems, Advances in Agroforestry Volumen 8, XVIII, 310 p.USA

25. Lagemann, L. \& Heuveldop, J. (1983). Characterization and evaluation of agroforestry systems: The case of Acosta-Puriscal, Costa Rica. In: Agro forestry Systems 1:101-115. P 15.

26. Lozano, A. (2009). Acceso al crédito en el sector cafetero colombiano. Ensayos sobre Economía Cafetera, $25,95-121$

27. La evaluación de los ecosistemas del milenio perspectivas y retos para la toma de decisiones. (2008). Ciudad de México. Recuperado de: http://www.millenniumassessment.org/documents/document.356.aspx.pdf

28. Montagnini, F. \& Nair, P.K.R. (2004). Carbon sequestration: An underexploited environmental benefit of agroforestry systems. In: Agroforestry Systems 61: 281-295.
29. Oelbermann, M; Voroney, R.P. \& Gordon, A.M. (2004). Carbon sequestration in tropical and temperate agroforestry systems: a review with examples from Costa Rica and southern Canada. In: Agriculture, Ecosystems and Environment 104: 359-377.

30. Ortiz, A., Riascos, L. \& Somarriba, E. (2008). Almacenamiento y tasas de fijación de biomasa y carbono en sistemas agroforestales de cacao (Theobroma cacao) y laurel (Cordia alliodora). Agroforestería en las Américas (CATIE) Volumen 46, 26 - 29, Costa Rica.

31. Ordoñez, C.M. (2013). Relación de la rentabilidad con la sostenibilidad en sistemas de producción tecnificados en fincas cafeteras de Pitalito (Huila).Tesis M.Sc. Universidad de la Amazonia. Colombia. p 92

32. Perfecto, I., Rice, RA., Greenberg, R. \& Van der Voort, ME. (1996). Shade coffee: a disappearing refuge for biodiversity. BioScience 46(8): 598-608.

33. Ruark G.A., Schoeneberger, M.M \& Nair P.K.R. (2003). Agroforestry-Helping to Achieve Sustainable Forest Management. UNFF (United Nations Forum for Forests) Intersessional.

34. Segura, M. \& Kanninen, M. (2002). Inventarios para estimar carbono en ecosistemas forestales tropicales. En: Orozco, L. y Brumer, C. Inventarios forestales para bosques latifolliados en América Central. Turrialba: CATIE, 2002. P. 202-222.

35. Schroeder, P. (1994). Carbon storage benefits of agroforestry systems. Agrofor. Syst. Vol 27, 89-97. Netherlands.

36. Suárez, D.A. (2002). Cuantificación y valoración económica del servicio ambiental almacenamiento de carbono en sistemas agroforestales de café de la Comarca Yassica Sur, Matagalpa Nicaragua. Maestría en Educación para el Desarrollo y la Conservación, CATIE, Turrialba, Costa Rica.

37. Schlegel, B., Gayoso, J. \& Guerra., J. (2001).Manual de procedimientos para inventarios de carbono en ecosistemas forestales. Universidad Austral de Chile. 117 pág.

38. Zanne, A.E., López, G., Coomes, D.A., Ilic, J., Jansen, S., Lewis, S.L., Miller, R.B., Swenson, N.G., Wiemann, M.C. \& Chave, J. (2009). Data from: Towards a worldwide wood economics spectrum.Dryad Digital Repository.

39. Viswanath, S., Kaushik, P.K. \& Prakasam, U., (1998). Biomass production and survival of Sesbania sesban in alley cropping. Forest, Farm and Community Tree Research Reports, vol. 3 pp. 59-61. 